\title{
An electron transparent proton detector for neutron decay studies
}

\author{
S. A. Hoedl ${ }^{\text {a) }}$ and A. R. Young ${ }^{\text {b) }}$ \\ Triangle University Nuclear Laboratory, Duke University, Durham, North Carolina 27708 \\ H. Ade \\ Department of Physics, North Carolina State University, Raleigh, North Carolina 27695
}

A. Lozano ${ }^{\text {) }}$

Department of Chemical Engineering, North Carolina State University, Raleigh, North Carolina 27695

(Received 21 October 2005; accepted 22 February 2006; published online 19 April 2006)

\begin{abstract}
We have developed an ultrathin $(<100 \mathrm{~nm})$, very strong polyimide foil which can span more than $6 \times 6 \mathrm{~cm}^{2}$ and is ideal for the fabrication of low energy proton detectors. We have produced a proton detector geometry in which protons incident on the foil with kinetic energies greater than about $25 \mathrm{keV}$ produce, on average, more than ten secondary electrons in a conversion crystal evaporated on the back face of the foil. These secondary electrons can be "postaccelerated" and counted in a variety of detectors. The polyimide foils are much more durable than carbon foils previously used in similar detection geometries. LiF was chosen as the conversion crystal, which is relatively insensitive to exposure to air, improving their secondary electron yield under typical operating conditions. In addition, we describe the operation of a very simple, small scale proton accelerator and detector testing chamber capable of providing up to $10 \mathrm{kHz}$ of beam with energies between 10 and $50 \mathrm{keV}$ onto a biased target with a maximum ion contamination of $0.5 \%$. (C) 2006 American Institute of Physics. [DOI: 10.1063/1.2186970]
\end{abstract}

\section{INTRODUCTION}

Measurements of the angular correlations between the decay products of free neutrons and the initial neutron spin direction provide high precision tests of the standard model without the nuclear structure corrections associated with measurements in higher mass nuclei. ${ }^{1}$ Recent progress in the development of cold neutron beam techniques and ultracold neutron (UCN) sources has made measurements of free neutron decay possible that are directly competitive with the super allowed $0^{+} \rightarrow 0^{+}$decays in low $Z$ nuclei, and has motivated a generation of experiments on neutron decay. ${ }^{1}$

A great deal of emphasis has been placed on measurements of the $\beta$ asymmetry in neutron decay because the detection of the $\beta$ particle is relatively straightforward. The physics reach of free neutron decay measurements can be greatly extended by the simultaneous detection of both the decay $\beta$ particle (782 keV end point energy) and the recoil proton (754 eV end point energy). ${ }^{2}$ Because angular correlations involving the $\beta$ particle and the proton have differing sensitivities to various extensions to the electroweak standard model, ${ }^{3}$ one can probe physics beyond the standard model with proton detection capability. In addition to these correlations, implementing simultaneous detection provides one with a powerful tool for eliminating one of the largest systematic uncertainties in previous $\beta$ asymmetry measurements: uncertainty in the absolute degree of neutron polar-

\footnotetext{
${ }^{a)}$ Present address: Center for Experimental Nuclear and Particle Astrophysics, University of Washington, Box 354290, Seattle, Washington 98195.

b) Also at Department of Physics, North Carolina State University, Raleigh, North Carolina 27695.

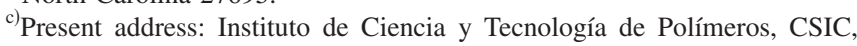
Juan de la Cierva 3, 28006-Madrid, Spain.
}

ization. By simultaneously measuring $\beta$ and proton asymmetries and taking their ratio, one determines a quantity which is independent of the neutron polarization to first order and can be used to extract the relevant physical parameters for neutron decay.

A scheme similar to the one we present here was attempted by the PERKEO II group utilizing carbon foils with $\mathrm{MgO}$ conversion crystals. ${ }^{4}$ Using this technique as a starting point, we have developed a detector technology which employs more durable and practical foils and utilizes a conversion crystal whose electron yield is not strongly degraded by exposure to air and whose performance is superior to $\mathrm{MgO}$ when implemented in practical experiments.

In this paper we present the construction and prototype tests of a proton conversion foil. In Sec. II, we outline the motivation for proton conversion foils as low energy proton detectors; next, some of the options we considered for foil substrates and conversion materials are presented (Secs. III and IV). In Sec. V we present measurements of the secondary yield. We then describe the proton accelerator and detector testing apparatus we constructed to evaluate our foils in Sec. VI. In Sec. VII we present calculations of the resultant proton detection efficiencies for our measured yields, and finally in Sec. VIII we present our conclusions.

\section{THE PROTON DETECTION TECHNIQUE}

The low energy of the recoil protons makes effective detection a technical challenge. With suitable acceleration, they can be detected with scintillators, ${ }^{3}$ silicon detectors, ${ }^{5}$ or microchannel plate detectors. ${ }^{2}$ Alternatively, the accelerated protons can be converted into secondary electrons. ${ }^{6,7}$

Our approach is to permit the recoil protons from $\mathrm{UCN}$ 


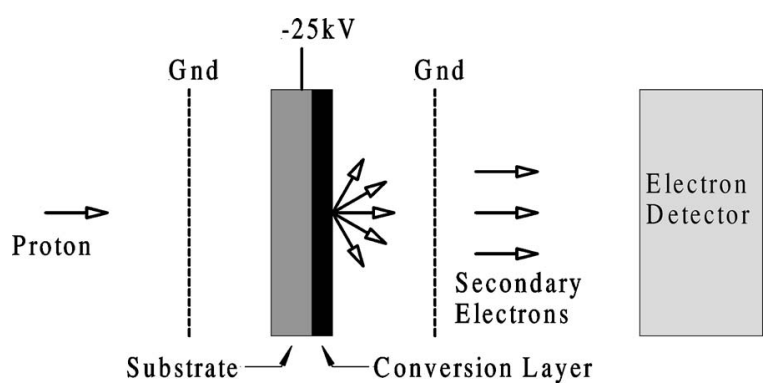

FIG. 1. A schematic of our proton detection technique. Two aluminum layers, one at the front face and one between the substrate and the conversion layer, serve to ensure electrical conductivity through the foil and are not shown here.

decay to drift through a fiducial decay volume and then pass a grounded grid (see Fig. 1). The protons are then accelerated into a very thin $(\sim 100 \mathrm{~nm})$, biased foil $(\sim-25 \mathrm{kV})$. The foil consists of a very strong substrate, coated with a high yield conversion crystal. As the protons exit the backside of the foil, they pass through a $\sim 10 \mathrm{~nm}$ thick layer of insulating crystal and liberate secondary electrons. These secondary electrons are then accelerated by the foil bias into a conventional electron detector: a gas counter, a plastic scintillator, or even a silicon detector. Electrons from neutron decay pass through the foil unhindered and can be detected by the same electron detector.

This technique allows one to use a $\beta$-decay spectrometer, such as the one already under construction by the UCNA collaboration at Los Alamos ${ }^{8}$ to measure the $\beta$ asymmetry, as a complete neutron decay detector. By placing the proton to electron conversion foils in front of the electron detectors, one can simultaneously detect and identify the electrons and protons from neutron decay.

\section{THE SUBSTRATE}

Carbon foils, used as substrates for nuclear physics targets, are a natural choice for the detector foil. Reference 4 reports the production of $8-22 \mu \mathrm{g} / \mathrm{cm}^{2}$ thick foils over square apertures of $13 \times 9.5 \mathrm{~cm}^{2}$. Unfortunately, these foils must be handled with extreme care. Table I lists other candidates and their relevant properties. We found that $6 \mathrm{~F} 6 \mathrm{~F}$

TABLE I. Selected properties of prospective substrate materials taken from Refs. 29-31 and www.goodfellow.com. The minimum thickness of polyimide and $6 \mathrm{~F} 6 \mathrm{~F}$ is from this research. The minimum thickness of polystyrene is from Ref. 12. Mylar® is biaxially oriented.

\begin{tabular}{lcccc}
\hline \hline Substrate & $\begin{array}{c}\text { Tensile } \\
\text { strength } \\
(\mathrm{MPa})\end{array}$ & $\begin{array}{c}\text { Elongation } \\
\text { at break } \\
(\%)\end{array}$ & $\begin{array}{c}\text { Min. } \\
\text { thick. } \\
(\AA)\end{array}$ & $\begin{array}{c}\text { Max. } \\
\text { temp. } \\
\left({ }^{\circ} \mathrm{C}\right)\end{array}$ \\
\hline Cellulose nitrate & 68 & 10 & 1000 & 85 \\
Polycarbonate & 64 & 100 & 500 & 115 \\
Polypropylene & 35 & 150 & $10^{4}$ & 90 \\
Polystyrene & 30 & 1.6 & 100 & 50 \\
Mylar® & 200 & 60 & 4000 & 115 \\
Polyimide & 121 & 8 & 600 & 250 \\
6F6F & 95.7 & 8 & 600 & 320 \\
\hline \hline
\end{tabular}

(synthesized in one of our laboratories) offered the most promise due to its tensile strength, temperature stability, and ease of thin-film manufacture.

6F6F (CAS No. 32036-79-6 or CAS No. 29896-40-0 also known as 6FDA-4,4'-6FDA) is a fluorinated polyimide which is synthesized through a condensation reaction between dianhydride and diamine monomers. (See Ref. 9 for an example of a synthesis process and see Ref. 10 for a summary of industry standard select properties, but note that our 6F6F probably derives its great strength from the high molecular weight synthesis process developed by one of the authors of this paper, Lozano.) $6 \mathrm{~F} 6 \mathrm{~F}$ is soluble in polar organic solvents, such as tetrahydrofuran (THF) or $\mathrm{N}$-methyl-2-pyrrolidone. The $\mathrm{H}_{2} \mathrm{O}$ contact angle for $6 \mathrm{~F} 6 \mathrm{~F}$ foils still on a silicon substrate was $83.5 \pm 0.2^{\circ}$, indicating that the foils are slightly hydrophilic. These properties enable a simple and inexpensive manufacture method of very thin 6F6F foils: spin coating.

Our manufacture process is as follows: We first mixed a $1 \%$ solution, by weight, of $6 \mathrm{~F} 6 \mathrm{~F}$ in high purity $\mathrm{THF}$ in a precleaned (through a plasma discharge process) $20 \mathrm{ml}$ borosilicate glass vial (I-CHEM C226-0020). The mixture was stirred with a teflon coated magnetic rod for $4 \mathrm{~h}$ and then allowed to sit over night. The mixture was then filtered twice through a $0.2 \mu \mathrm{m}$ polytetrafluoroethylene (PTFE) membrane with a glass microfiber prefilter in a polypropylene housing (Whatman AV125EORG) and then stored in a second precleaned vial.

We spun our foils onto $100 \mathrm{~mm}$ diameter silicon wafers. The polarity of the silicon ( $n$ or $p$ ) and crystal orientation of the surface were not critical. The surface of the wafers, as received from the manufacturer, was extremely hydrophobic. THF, a polar solvent, will not wet a hydrophobic surface, and thus, spin coating on the silicon wafers as delivered was not possible. We therefore treated the silicon surface with a mercury discharge lamp in ambient atmospheric conditions for $1 \mathrm{~h}$. The UV light, in combination with the ozone generated by the UV interaction with atmospheric $\mathrm{O}_{2}$, terminated the surface with $\sim 10 \AA$ of hydrophilic $\mathrm{SiO}_{2}$ and removed any residual hydrocarbon contamination. ${ }^{11}$ Before spin coating, the wafer was given a rinse with de-ionized water and blown dry with filtered research grade $\mathrm{N}_{2}$. The rinse step ensured that at least a monolayer of $\mathrm{H}_{2} \mathrm{O}$ covered the silicon surface. The $6 \mathrm{~F} 6 \mathrm{~F} / \mathrm{THF}$ solution was extracted from the storage vial into a $5 \mathrm{ml}$ polyethylene/polypropylene syringe through a B-D 20G $11 / 2$ precision glide needle. $3 \mathrm{ml}$ of the solution was then deposited through the needle onto the silicon wafer which was then immediately spun at $1000 \mathrm{rpm}$ for $30 \mathrm{~s}$. The surface tension of the polymer/solvent solution, in combination with the adhesion (or wetting) of the solution to the substrate, balances the centrifugal force of the spinning action to produce a layer of uniform thickness. The thickness is a function of the polymer concentration in the solvent solution and the speed of rotation. A theoretical description of the process for polystyrene thin films is presented in Ref. 12.

Following the above surface treatments, removal of the $6 \mathrm{~F} 6 \mathrm{~F}$ foil from the silicon substrate was very easy. Held at the edges by hand, we slowly dipped the silicon wafer into a tank of water at a slight angle to the surface of the water. The 
TABLE II. The results of energy loss measurements through two $6 \mathrm{~F} 6 \mathrm{~F}$ foils We assume a mass density of $1.47 \mathrm{~g} / \mathrm{cm}^{3}$ from Ref. 10 . The uncertainty in the areal density is dominated by the uncertainty of the proton stopping power in $6 \mathrm{~F} 6 \mathrm{~F}$.

\begin{tabular}{cccc}
\hline \hline Foil & $E_{\text {loss }}(\mathrm{keV})$ & Areal density $\left(\mu \mathrm{g} / \mathrm{cm}^{2}\right)$ & Thickness $(\AA)$ \\
\hline 1 & 7.5 & $11.5 \pm 1.5$ & $780 \pm 100$ \\
2 & 5.6 & $8.6 \pm 1.0$ & $580 \pm 75$ \\
\hline \hline
\end{tabular}

$6 \mathrm{~F} 6 \mathrm{~F}$ immediately parted from the wafer and floated onto the surface of the water. When sufficient polymer had floated off, we could use our other hand to take hold of the wafer under the surface of the water and continue parting the foil from the silicon. Once completely removed, the wafer was allowed to drop to the bottom of the water tank. We gently removed the $6 \mathrm{~F} 6 \mathrm{~F}$ foil from the surface of the water by carefully positioning an edge of an appropriate frame next to the foil. Equally carefully, the edge of the foil was made to contact an edge of the frame, whereupon the foil would adhere to the frame. The frame was then slowly lifted from the water, perpendicular to the water surface, such that the foil spanned the opening area of the frame. The frame/foil combination was then heated at a moderate temperature $\left(\sim 30{ }^{\circ} \mathrm{C}\right)$ until the water evaporated, and the foil relaxed in the frame to achieve a taut surface. The smallest foils made via this method were on circular frames with an open diameter of $0.64 \mathrm{~cm}$. The largest foils were circular with a $10 \mathrm{~cm}$ diameter and mounted on square frames with an open side of length $5.6 \mathrm{~cm}$. The strength of the resulting foils is impressive. In contrast to carbon foils, one can walk, talk, and breath on $6 \mathrm{~F} 6 \mathrm{~F}$ foils in open air without breaking them. They also routinely survived pump down in vacuum systems without requiring a lengthening of the roughing pump time.

We measured the mass thickness of the $6 \mathrm{~F} 6 \mathrm{~F}$ foils by measuring the energy loss of a $45 \mathrm{keV}$ proton beam through the foil. The proton accelerator is described in Sec. VI. The $6 \mathrm{~F} 6 \mathrm{~F}$ foil was mounted so that it could be moved out of the beam, thus allowing the $50 \mathrm{~mm}^{2}$ silicon detector to be calibrated by the proton beam itself. Using a SRIM2000 simulation, ${ }^{13}$ we estimated the energy loss of a $45 \mathrm{keV}$ proton beam through the $6 \mathrm{~F} 6 \mathrm{~F}$ foil as a function of the foil thickness, taking into account the angular acceptance of the $50 \mathrm{~mm}^{2}$ silicon detector $(\Delta \theta=0.02 \mathrm{rad})$. With this method, we measured the thickness of two foils to be $11.5 \pm 1.5$ and $8.6 \pm 1.0 \mu \mathrm{g} / \mathrm{cm}^{2}$. The results are summarized in Table II. The two foils were manufactured using the same technique as described above, by the same experimenter, on the same day, indicating that the foil thickness is not easy to precisely reproduce.

\section{THE CONVERSION CRYSTAL}

Typical secondary electron emitters include metal oxides and alkali halides. Note that the yield from 6F6F, as that of most plastics, is relatively poor at, on average, 1 secondary per incident proton. In selecting a crystal, the overriding constraints are that the crystal must be able to be easily and cheaply deposited onto $6 \mathrm{~F} 6 \mathrm{~F}$, and must be able to survive exposure to the ambient atmosphere. Promising candidates
TABLE III. The vacuum evaporation deposition parameters for the prototype detectors.

\begin{tabular}{cccccc}
\hline \hline Foil & $\begin{array}{c}\text { pressure } \\
(\text { Torr })\end{array}$ & $\begin{array}{c}\mathrm{Al} \\
\text { Thick. }(\AA)\end{array}$ & $\begin{array}{c}\text { Al rate } \\
(\AA / \mathrm{\AA})\end{array}$ & $\begin{array}{c}\text { Crystal } \\
\text { thick. }(\AA)\end{array}$ & $\begin{array}{c}\text { Crystal } \\
\text { rate }(\AA / \mathrm{s})\end{array}$ \\
\hline LiF & $1.0 \times 10^{-6}$ & 110 & 1 & 206 & 0.05 \\
CsI & $8.6 \times 10^{-7}$ & 64 & 6 & 197 & 40 \\
\hline \hline
\end{tabular}

include $\mathrm{LiF}, \mathrm{Al}_{2} \mathrm{O}_{3}$, hexatriacontane and diamond due to their high yield and surface stability. Note that although $\mathrm{MgO}$ has been considered in the past for similar applications, its surface is more reactive than that of $\mathrm{NaCl}$ to both $\mathrm{H}_{2} \mathrm{O}$ and $\mathrm{CO}_{2} ;{ }^{14}$ the secondary yield is not stable to exposure to these gases. 15

$\mathrm{Al}_{2} \mathrm{O}_{3}$ has the distinct advantage that its surface is robust to atmospheric exposure. Alumina ceramic has an electron impact induced secondary yield between 5 and 7 at an incident energy of $1 \mathrm{keV}$. At the same energy, a sapphire crystal has a yield of 10.1. ${ }^{16}$ Deposition of $\mathrm{Al}_{2} \mathrm{O}_{3}$ on $6 \mathrm{~F} 6 \mathrm{~F}$ foils, however, may be difficult. Of the alkali halides, $\mathrm{LiF}$ has the most stable surface. At an incident energy of $600 \mathrm{eV}$, a vacuum cleaved crystal has a peak yield of $7 .^{15}$ At an incident energy of $1.1 \mathrm{keV}$, a vacuum evaporated thin film has a peak yield of $8 .{ }^{17}$ Brief exposure of $\mathrm{LiF}$ to water vapor appears to reduce the electron affinity and increase the secondary yield. ${ }^{18}$ The principal advantage of LiF is the ease of thermal vacuum deposition. In this paper, we explore how well LiF will work as a conversion crystal. For comparison we also have tested CsI, since it is a common photocathode material for $\mathrm{x}$-ray and UV photon detectors. ${ }^{19}$

In passing, we note two additional materials which we have identified, warranting further research due to their negative electron affinity (NEA): Hexatriacontane ${ }^{18}$ (HTC, $n$ - $\mathrm{C}_{36} \mathrm{H}_{74}$, CAS No. 630-06-08) and deuterium or cesium terminated diamond-like-carbon. ${ }^{20-22}$ NEA materials present the distinct advantage that low energy secondary electrons created in the crystal which reach the material surface are "pushed" out of the crystal instead of reflected back into the material by the more typically positive work function. In addition, these materials have long secondary electron attenuation lengths, meaning that secondaries created deep in the material have a greater chance of reaching the surface. From a practical perspective, both HTC and hydrogen terminated diamond are resilient to atmospheric exposure, and stable in UHV conditions. HTC can also be readily vacuum evaporated.

\section{MEASURED SECONDARY YIELD}

We constructed proton conversion foils in the following manner. First, we manufactured $67.5 \mathrm{~nm}$ thick 6F6F films supported on stainless steel frames with a circular aperture of $6.3 \mathrm{~mm}$. Next, we evaporated $\mathrm{Al}$ onto both sides of the 6F6F. The Al provides electrical conductivity to the foil, necessary for continuous proton conversion. Without breaking vacuum, we next evaporated the conversion crystal. Table III summarizes the deposition parameters. The deposition vacuum was maintained through a $\mathrm{LN}_{2}$ cold trap by an oil diffusion pump, filled with Santovac 5, backed by an oil-based rough- 


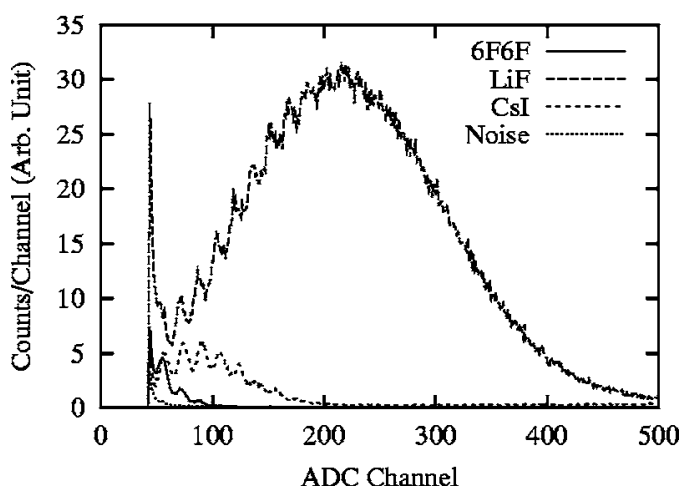

FIG. 2. The pulse height spectrum in the silicon detector when prototype uncoated and CsI and $\mathrm{LiF}$ coated foils were exposed to a $45 \mathrm{keV}$ proton beam. The first peak in the spectrum was due to electronic noise; the additional distinct peaks in the spectrum represent additional electrons liberated by the foil and detected by the silicon detector. Each secondary had an energy of $20 \mathrm{keV}$. The absolute normalization of the spectrum are scaled for clarity.

ing pump. The aluminum source was a piece of $99.99 \%$ pure aluminum wire in a tantalum boat. The LiF source was a 99.9\% pure powder in a tantalum boat. The CsI source was a single crystal, also in a tantalum boat. All sources were properly out-gassed before evaporation. Immediately following the evaporation, all foils had a mirrorlike quality. After 5 to $10 \mathrm{~min}$ of exposure to air, however, the CsI foil rapidly acquired an opaque blue appearance. After a few hours of exposure to the atmosphere, the $\mathrm{LiF}$ foils became crinkly. Once placed in an evacuated chamber, however, the LiF foils regained their mirrorlike appearance, indicating that either the $6 \mathrm{~F} 6 \mathrm{~F}$ or the $\mathrm{LiF}$ layer absorbed atmospheric gases or water vapor. The CsI foil never lost the blue color, indicating a permanent change most likely due to water adsorption.

Each detector was exposed to a proton beam with a kinetic energy between 20 and $45 \mathrm{keV}$ and at a rate between 100 and $1000 \mathrm{~Hz}$. At each energy, the proton detectors were biased to $-20 \mathrm{kV}$. The liberated secondary electrons were accelerated into a silicon detector with an efficiency of nearly $100 \%$ (see the discussion at the end of this paper). The pulse height histogram of the pulses from the silicon detector was then recorded with an Ortec PC-based multi-channel analyzer. The extraction and focus voltages of the electrode structure were adjusted to maximize the mean of the pulse height distribution (see discussion at end of this paper). Figure 2 presents representative spectra for an uncoated $6 \mathrm{~F} 6 \mathrm{~F}$ foil, a CsI coated foil, and a LiF coated foil, at an incident proton energy of $45 \mathrm{keV}$. The first peak is due to electronic noise and proton energy deposition in the silicon detector. We hypothesize that the first electron peak is near analog-todigital converter (ADC) channel 50. The 6F6F itself generates few secondary electrons. The CsI coated foil also generates relatively few SE; yet, the CSI spectrum also has a very long tail. We hypothesize that the tail may be due to the scintillating properties of CsI. The protons may be creating scintillator light, which, in turn, excites the silicon detector or liberates secondaries from other parts of the vacuum system, which, in turn, are detected by the silicon detector.

Focusing now on LiF coated foils, interpretation of the secondary electron spectrum is complicated by two factors:

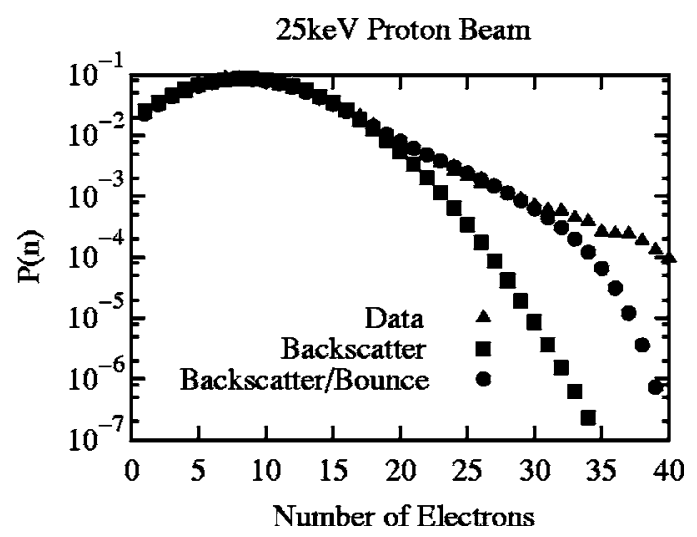

FIG. 3. The rebinned, measured electron spectra for $25 \mathrm{keV}$ incident proton energy plotted with the two nonlinear fits accounting for electron backscatter alone and electron backscatter with proton reflection from the accelerating fields.

electron backscatter from the silicon detector itself and proton field reflection from the electric fields in the focusing electrodes (also referred to as a proton "bounce" in this paper). $20 \mathrm{keV}$ electrons have a $17 \%$ chance of backscatter from silicon; scattered electrons have a much lower chance of being seen in the detector and thus reduce the observed secondary yield. Protons which exit the 6F6F foil will decelerate in the electric fields; if their energy is low enough, they will turn around, hit the 6F6F foil, and generate another secondary electron shower. Using a SRIM2000 calculation to estimate the proton energy and direction after passage through the foil, combined with an electric field transport algorithm to trace the path of the protons in the electrode structure, we estimate that only $9 \%$ of the protons for the $25 \mathrm{keV}$ test returned to the foil and generated secondary electrons that could be seen. For the 35 and $45 \mathrm{keV}$ test cases, we estimated that no protons could return to the foil. The spectra presented in Fig. 2 are thus dominated by single proton-foil interactions.

We analyzed the spectrum in the following manner. First, a Gaussian fit was applied to the first 11 electron peaks in the spectrum to identify the electron number as a function of the ADC channel. On the basis of these fits, the spectrum was rebinned in units of the electron number to simplify the analysis. One can show that electron backscatter from the surface of our Si detector changes the observed probability distribution according to

$$
P_{\mathrm{obs}}(N)=\sum_{n=N}^{\infty} \frac{n !}{N !(n-N) !}(1-\eta)^{N} \eta^{n-N} P_{\mathrm{lib}}(n),
$$

where $N$ is the number of secondary electrons observed in the silicon detector, $\eta$ is the probability that a $20 \mathrm{keV}$ electron will backscatter $(0.17)$, and $P_{\mathrm{lib}}(n)$ is the probability that $n$ electrons will be liberated by one incident proton. We have considered two possible forms of $P_{\text {lib }}(n)$ : first, neglecting the possibility of a proton field reflection, we let

$$
P_{\mathrm{lib}}=e^{-(n-\mu)^{2} / 2 \sigma^{2}} .
$$

Second, to account for a proton field reflection, we let 
TABLE IV. The fit parameters for the prototype $\mathrm{LiF}$ foil.

\begin{tabular}{ccccccc}
\hline \hline \multirow{2}{*}{$\begin{array}{c}\text { Incident } \\
\text { energy }(\mathrm{keV})\end{array}$} & \multicolumn{2}{c}{ Backscatter } & & \multicolumn{3}{c}{ Proton bounce } \\
\cline { 2 - 3 } \cline { 5 - 7 }$n$ & $\mu$ & $\sigma$ & & $\mu$ & $\sigma$ & $b$ \\
\hline 25 & 10.3 & 5.6 & & 10.0 & 5.2 & 0.08 \\
35 & 12.2 & 6.1 & & 11.9 & 5.8 & 0.07 \\
45 & 13.3 & 6.7 & & 13.1 & 6.6 & 0.03 \\
\hline \hline
\end{tabular}

$$
P_{\mathrm{lib}}(n)=(1-b) P(n)+b \sum_{j=0}^{j=n} P(j) P(n-j),
$$

where $b$ is the probability that a proton will return to an active area of the proton detector, and $P(n)$ is the probability that a single proton traverse or impact will liberate $n$ electrons. In this second case, $P(n)$ is chosen to be

$$
P(n)=e^{-(n-\mu)^{2} / 2 \sigma^{2}} .
$$

For the two forms of $P_{\text {lib }}(n), \mu, \sigma$, and $b$, are estimated on the basis of a nonlinear least-squares fit to the rebinned data. Figure 3 plots the $25 \mathrm{keV}$ rebinned data and the two fits. Table IV lists the fit parameters at each proton energy. Notice that the proton field reflection fit accounts for the distribution tail much better than the backscatter fit alone suggesting that we saw evidence for proton field reflection. The proton return probability $b$ is consistent with our expectations at $25 \mathrm{keV}$, but not at 35 or $45 \mathrm{keV}$. Other rare events may contribute to the probability tail, such as proton impact on the focusing electrode structure, an occurrence that would mimic a proton field reflection. Note that the difference in the estimated yield is less than $3 \%$ between the two fitting cases. Note also that to an accuracy of $5 \%$, the average yield can be estimated on the basis of a Gaussian fit to the observed spectrum: $\mu=\mu_{\mathrm{obs}} /(1-\eta)$. The width of the Gaussian distribution is much broader than expected from counting statistics alone: $\sigma=1.7 \sqrt{\mu}$. This is probably due to energy and angle straggle of the protons through the foil.

We performed a simple aging test for the LiF prototypes in the following manner. Two prototype detectors, each coated with $50 \AA$ of $\mathrm{Al}$ followed by $60 \AA$ of $\mathrm{LiF}$ at a deposition rate of $0.25 \AA / \mathrm{sec}$, were manufactured simultaneously. The secondary yield of one foil at $45 \mathrm{keV}$ was immediately measured. After one week storage in a container filled with ambient air, the yield of the other foil at $45 \mathrm{keV}$ was measured. Figure 4 plots the spectra. The average yield (corrected for electron backscatter) fell from 12.5 to 6.5 . The reduced variance, $\sigma / \sqrt{\mu}$ increased from 1.7 to 2.2. Clearly, despite the stability of the LiF surface, care must be taken for long term storage of the $\mathrm{LiF}$ foils.

\section{A SIMPLE PROTON ACCELERATOR}

To test the prototype conversion foils and obtain the data presented in Sec. VI, we built a simple proton accelerator (see Fig. 5) capable of providing protons at a rate of $0-10 \mathrm{kHz}$ with a kinetic energy up to $50 \mathrm{keV}$ and a maximum ion contamination of less than $0.5 \%$. The accelerator consisted of the following elements in beam order: a $\mathrm{H}_{2}$ variable leak and gas handling system, a $100 \mathrm{~W}$ light bulb fila-

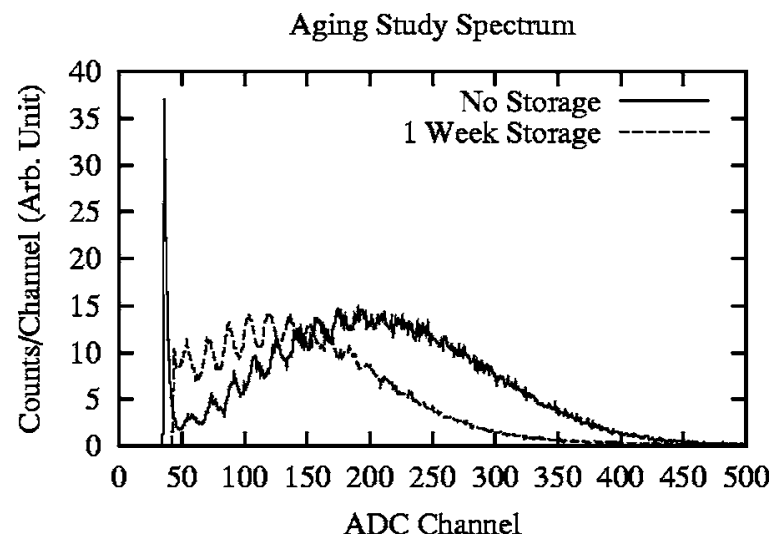

FIG. 4. The spectra from a LiF prototype tested immediately by a $45 \mathrm{keV}$ proton beam and tested after one week exposure to atmospheric conditions.

ment and associated power supply, a $60 \mathrm{kV}$ electrostatic acceleration column, a dry pumping station, an electromagnet to separate the ion species, and a series of apertures to define the entrance and exit path of the beam. Note that due to the very low count rate requirements, neither steering nor focusing elements were necessary or used. Each side of the acceleration column could be biased independently of the other to $\pm 40 \mathrm{kV}$. This bias scheme allowed the proton energy, determined by the potential difference across the acceleration column, to be different than the foil bias. Except for the proton source, all electronics, such as pumps, the magnet, and the electron detectors were operated at ground potential. The end of the accelerator was connected to a detector testing chamber.

Protons were liberated from $\mathrm{H}_{2}$ by the ionizing action of electrons emitted by a hot, $100 \mathrm{~W}$ light bulb filament suspended between two feedthroughs in the middle of a $5 \mathrm{~cm}$ long, $11 \mathrm{~cm}$ OD Al spool piece (see Fig. 6). The upstream end of this spool piece was closed off by a brass plate, opened at the edges for vacuum pump out. The downstream end was limited by an $\mathrm{Al}$ aperture, directly connected to the acceleration column. The exit aperture and filament were held at a fixed potential by a high voltage power supply. The cylindrical walls and brass plate were positively biased with a $300 \mathrm{~V}$ battery with respect to the filament and exit aperture. The bias served to extract electrons from the filament and provide sufficient energy for $\mathrm{H}_{2}$ ionization in addition to focusing the ions through the exit aperture. The filament was powered by a variac connected to a $50 \mathrm{kV}, 250 \mathrm{~W}$, isolation transformer.

Following the acceleration column, the ions passed

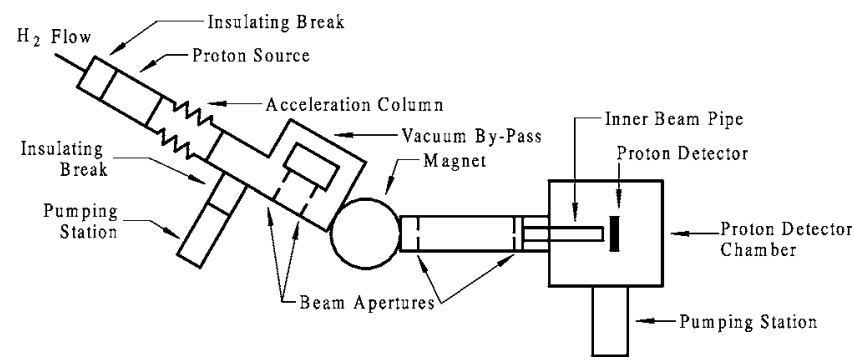

FIG. 5. A schematic of the proton accelerator (secondary electron detection apparatus not shown); not to scale. 


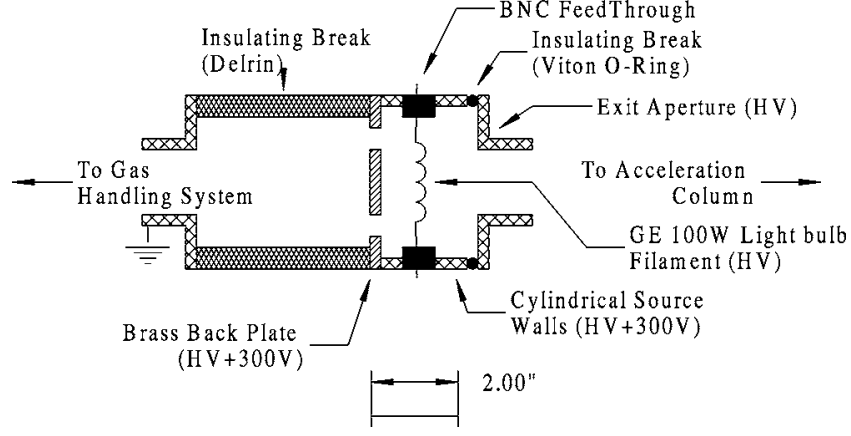

FIG. 6. A scale schematic of the proton source. The filament and exit aperture could be biased up to $\pm 40 \mathrm{kV}$ ( $\mathrm{HV}$ in the diagram).

through two $3 \mathrm{~mm}$ diameter circular apertures punched in a $0.25 \mathrm{~mm}$ thick tantalum foil placed $30 \mathrm{~cm}$ apart at a sufficient distance from the bending magnet to ensure that the fringe field from the bending magnet was less than the Earth's field. After the apertures, the protons passed through a $15 \mathrm{~cm}$ diameter, $2.5 \mathrm{~cm}$ high, cylindrical bending magnet chamber by making a $30^{\circ}$ turn. The magnet chamber, biased at up to $+40 \mathrm{kV}$, was insulated from the grounded magnet pole tips by a $6 \mathrm{~mm}$ thick sheet of ultra high molecular weight (UHMW) polyethylene. UHMW polyethylene has the highest known dielectric strength $(2300 \mathrm{~V} / \mathrm{mil})$ of the commercially available plastics. After the bend, the ions traveled through another two $3 \mathrm{~mm}$ diameter apertures located $100 \mathrm{~cm}$ apart.

\section{A. Tests of the proton accelerator}

At a fixed proton source pressure $\left(5 \times 10^{-5}\right.$ Torr $)$, filament power $(5 \mathrm{~W})$, and acceleration potential $(40 \mathrm{kV})$, we recorded the count rate in a channeltron detector, positioned immediately downstream from the last aperture, as a function of the magnetic field in the bending magnet. A representative scan is plotted in Fig. 7. By also introducing He gas, we were able to positively identify four peaks due to $\mathrm{H}_{2}: \mathrm{H}^{+}, \mathrm{H}_{2}^{+}, \mathrm{H}_{3}^{+}$, and a peak due to the dissociation of $\mathrm{H}_{2}^{+}$into $\mathrm{H}$ and $\mathrm{H}^{+}$. Assuming a circular path in the bending magnet, and an equal sharing of momentum in the dissociation case, one would expect the magnetic field of the peaks

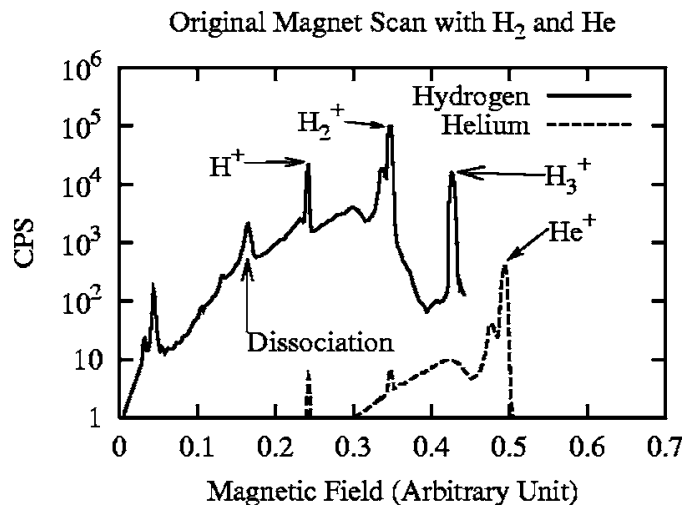

FIG. 7. Count rate in a Channeltron detector, as a function of the field in the bending magnet for hydrogen and helium gas. The origin of the lowest field, unlabeled peak remains unknown. The He gas case has been scaled down by a factor of 1000 for greater clarity.

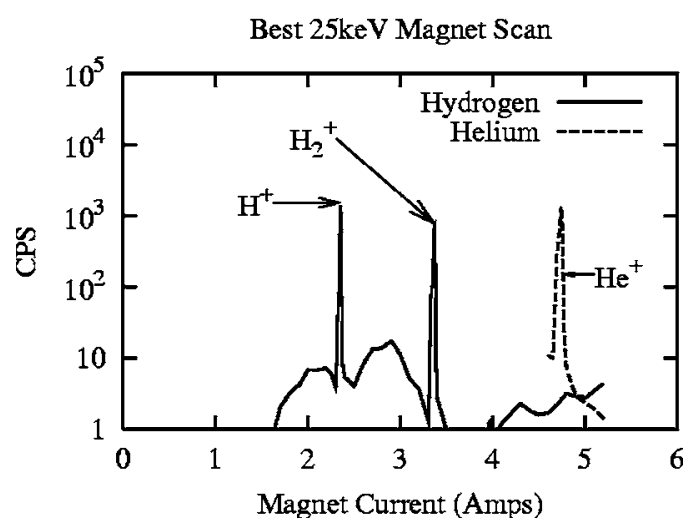

FIG. 8. An example of the count rate in the Channeltron detector as a function of the magnet current when $\mathrm{H}_{2}$ was not continuously introduced into the accelerator.

$\mathrm{H}_{\mathrm{dis}}: \mathrm{H}^{+}: \mathrm{H}_{2}^{+}: \mathrm{H}_{3}^{+}: \mathrm{He}^{+}$to be in the ratio $1 / \sqrt{2}: 1: \sqrt{2}: \sqrt{3}: 2$. We find the ratio to be $0.682: 1: 1.43: 1.76: 2.04$. The deviation from the expected ratio is less than $3 \%$.

We realized the best performance when hydrogen gas was not leaked into the vacuum. See Fig. 8 for a scan at $25 \mathrm{keV}$. The operation proceeded as follows: first, we flooded the vacuum system with $\mathrm{H}_{2}$ up to a pressure of 1 $\times 10^{-3}$ Torr. We then pumped out the $\mathrm{H}_{2}$ to a pressure of 1 $\times 10^{-5}$ Torr before operating the accelerator. When the accelerator was used continuously, $\mathrm{H}_{2}$ "treatments" were needed approximately once a day. A very conservative limit on the ion species contamination can be determined by dividing the peak height by the value of the lowest trough at fields above the $\mathrm{H}^{+}$peak. We put an upper bound on the ion contamination of $1.2 \%$ at $10 \mathrm{keV}, 0.3 \%$ at $25 \mathrm{keV}$, and $0.1 \%$ at $40 \mathrm{keV}$.

\section{B. The detector testing chamber}

We built a detector testing chamber (see Fig. 9) which had four critical elements: an inner beam pipe to carry the beam pipe potential from outside the detector chamber

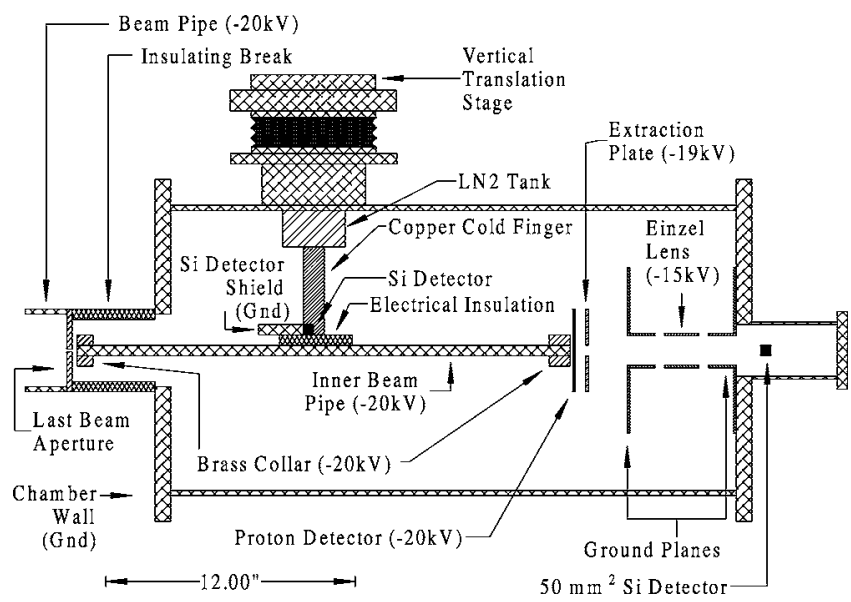

FIG. 9. A cross section schematic of the inside of the detector chamber. For clarity, structural elements are not shown. These include support and guide rails for the inner beam pipe, the detector translation stage, insulating supports for the focus electrodes, and the mounting fixtures of the $50 \mathrm{~mm}^{2}$ silicon detector. Vacuum pumps, view ports, and electrical feedthroughs are also not shown. 


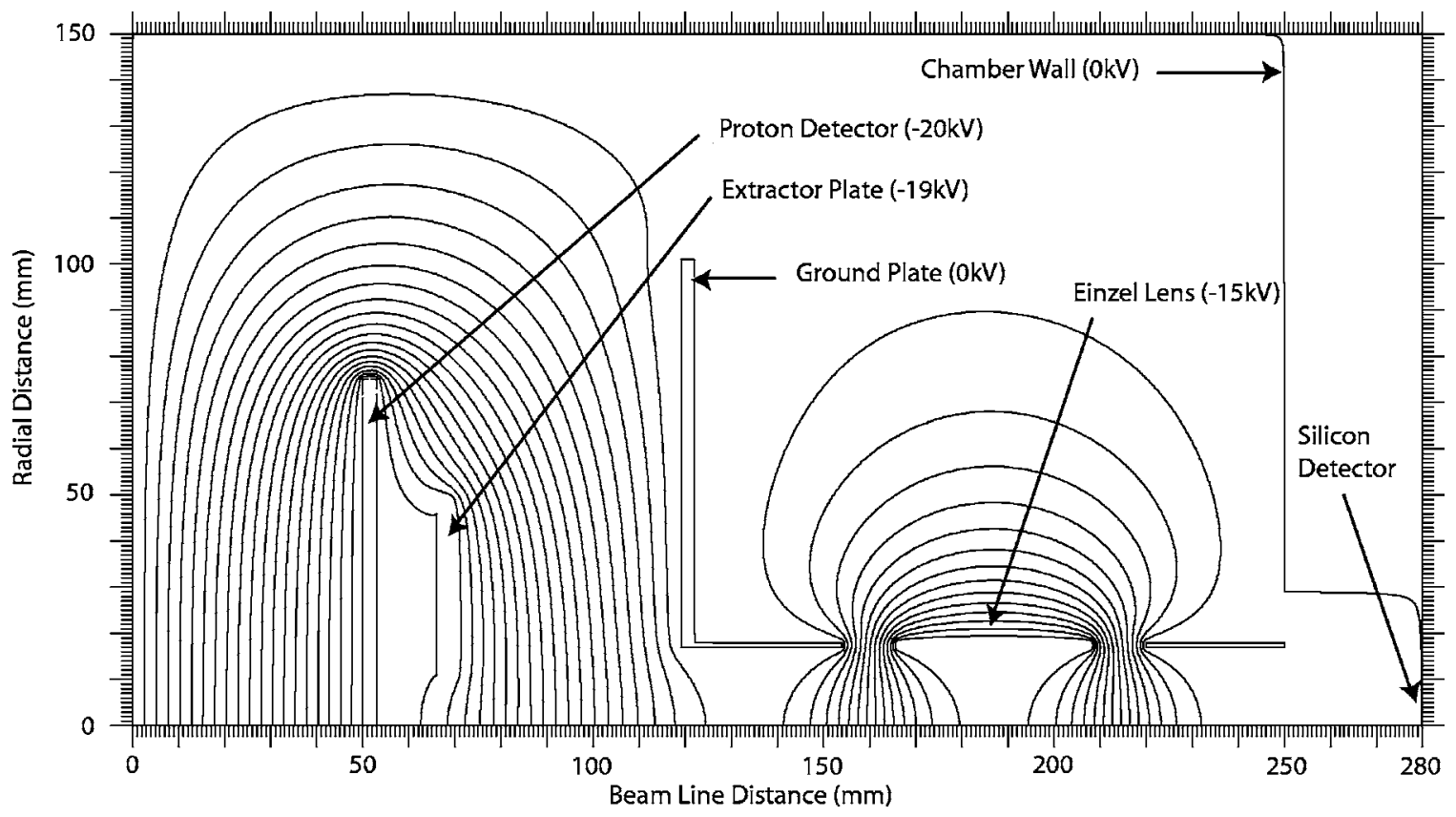

FIG. 10. A cutaway view of the equipotential surfaces near the focus elements as calculated by RELAX3D. Each equipotential surface represents 5\% of the potential of the proton foil (typically $-20 \mathrm{keV}$ ). The inner beam pipe, located immediately to the left of the proton detector, and the $200 \mathrm{~V}$ grid used for larger detection foils, are

through the inside of the chamber to the surface of the biased foil, a vertically translatable silicon detector $\left(25 \mathrm{~mm}^{2}\right.$ active area) to monitor the proton beam quality, a horizontal and vertical translation stage to scan the spatial response of the foils, and electrode elements to focus secondary electrons created by the conversion foil into another silicon detector $\left(50 \mathrm{~mm}^{2}\right.$ active area) located at the end of the chamber. The $25 \mathrm{~mm}^{2}$ detector was mounted on a copper cold finger connected to a $\mathrm{LN}_{2}$ dewar in the vacuum system. Brass collars at the ends of the inner beam pipe minimized beam defocusing by ensuring that electric potential gradients were far from the center of the beam path. The chamber could achieve an ultimate pressure of $5 \times 10^{-7}$ Torr.

\section{Secondary electron focusing elements}

The energy and angle distribution of secondaries produced by proton passage through $\mathrm{LiF}$ or CsI is unknown. For electron ${ }^{23}$ and ion ${ }^{24}$ impact on $\mathrm{Al}$, a $\cos \theta$ weighted distribution has been observed. X-ray impact on LiF gives a peak secondary energy of $\sim 3 \mathrm{eV}$ and a maximum secondary energy of $16 \mathrm{eV} .^{25}$ It is expected that the energy and angle distribution of the secondaries is independent of the type or energy of the incident radiation. ${ }^{26}$ We designed the electrode structure to optimally focus secondaries produced in a $3 \mathrm{~mm}$ radius circle (twice the radius of the final beam aperture) with a $\cos \theta$ weighted angular distribution with energies between 0.1 and $50 \mathrm{eV}$ into the $50 \mathrm{~mm}^{2}$ silicon detector.

For a small diameter $-20 \mathrm{kV}$ biased foil, our design consisted of an extraction plate (biased to $-19 \mathrm{kV}$ ), which ensured optimal collection and prefocusing of the secondary electrons, an acceleration region, which accelerated the electrons to their full energy, and an Einzel lens, consisting of a ground cylinder, a biased cylinder $(-15 \mathrm{kV})$, and then another ground cylinder, which provided the final focus. A labeled cutaway view of the equipotential surfaces is presented in Fig. 10. The trajectory path of 0.1 and $50 \mathrm{eV}$ electrons is presented in Fig. 11. A Monte Carlo simulation of the transport of $10^{4}$ electrons calculated a collection efficiency of $100 \%$ for both 0.1 and $50 \mathrm{eV}$ electrons. For larger foils, we added a $2 \mathrm{~mm}$ pitch grid biased at $+200 \mathrm{~V}$ with respect to the foil between the foil and the extraction plate. This grid
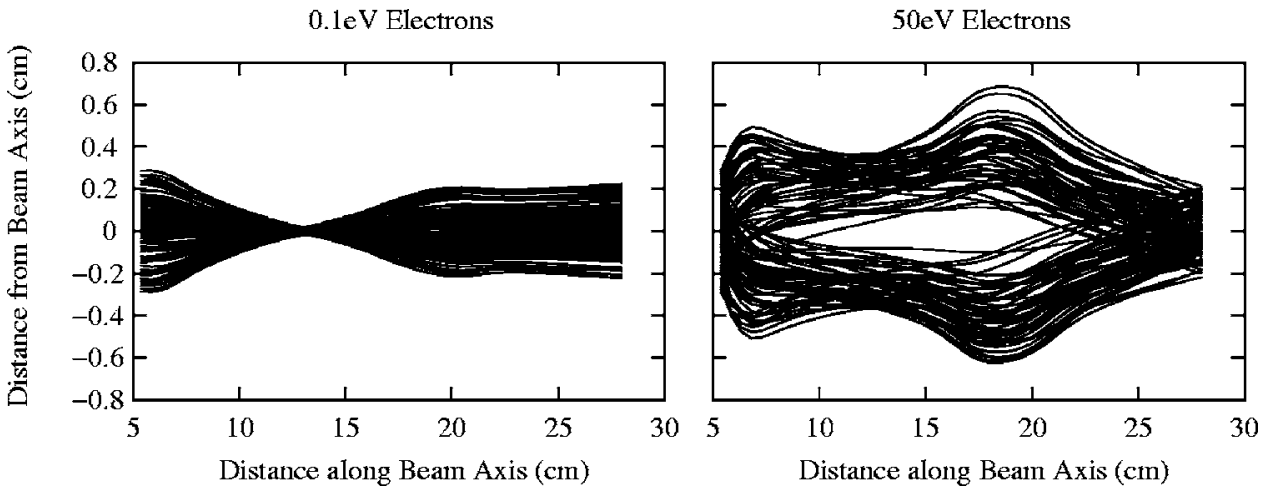

FIG. 11. The trajectory of 100 secondary electrons emitted from the proton foil at 0.1 and $50 \mathrm{eV}$. Note that the active area of the silicon detector was between $\pm 0.4 \mathrm{~cm}$ from the beam center and along the beam axis at $28 \mathrm{~cm}$. These simulations indicate the electrode structure had a collection efficiency of $100 \%$. 


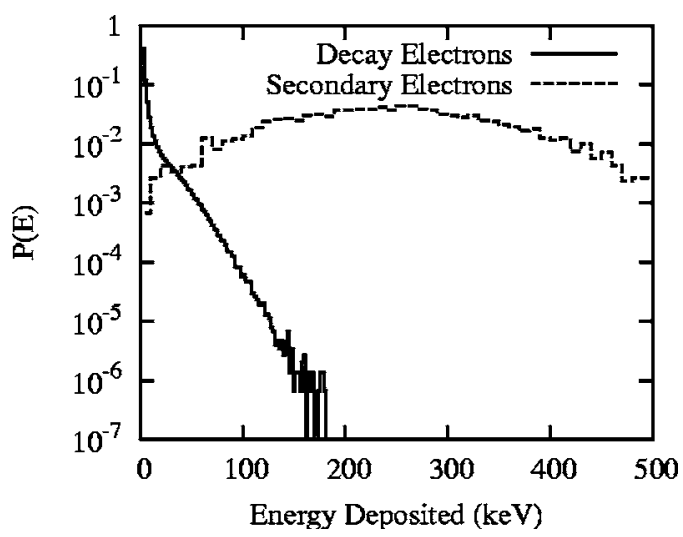

FIG. 12. A Monte Carlo energy deposition histogram in the MWPC due to secondary electrons from the proton conversion foil and due to electrons from neutron decay.

prevented larger foils from breaking due to the electrostatic force between the foil and the extractor plate.

\section{SIMULATED PROTON COUNTING EFFICIENCIES}

Any electron detector could be used to observe secondary electrons (SEs) from the proton conversion foil; a multiwire proportional counter (MWPC), however, offers perhaps the simplest way to distinguish $\beta$-decay electrons from secondary electrons. With a series of Monte Carlo simulations we have studied the expected response. The model MWPC consists of a $6 \mu \mathrm{m}$ thick mylar entrance window followed by a $2.0 \mathrm{~cm}$ thick active region filled with 100 Torr of neopentane

$\left(\mathrm{C}_{5} \mathrm{H}_{12}, \quad \rho=3.8 \times 10^{-4} \mathrm{~g} / \mathrm{cm}^{3}\right)$ and a $6 \mu \mathrm{m}$ thick mylar MWPC exit window. Neopentane was chosen as a prospective MWPC fill gas due to its high ion pair yield (estimated to be 1 ion pair/ $88 \mathrm{eV}$ deposited ${ }^{27}$ ) and low average $Z$. For analysis simplicity, we assume that the ion collection efficiency in the MWPC is $100 \%$.

We simulated the impact of 3000 protons on a $30 \mathrm{kV}$ biased conversion foil backed by the model MWPC. For each proton impact on the conversion foil, the number of liberated secondaries was sampled from a Gaussian distribution with a mean of 20 and a standard deviation of 7.6, consistent with the measured statistical distribution. Using a PENELOPE simulation, ${ }^{28}$ the total energy deposition in the active region of the MWPC was modeled for this number of normal incident $30 \mathrm{keV}$ electrons. For comparison, the energy deposition by electrons from $\beta$ decay in the UCNA experiment was also modeled with PENELOPE. Note that this simulation includes the effect of a magnetic field expansion region in the decay spectrometer which parallelizes the electron trajectories and reflects backscatter electrons back into the MWPC. Figure 12 plots the results of these simulations. We find that with an energy deposition threshold of 75,100 , and $125 \mathrm{keV}$, protons will be detected with an efficiency of $97 \%, 94 \%$, and $90 \%$ respectively. If a $125 \mathrm{keV}$ energy cut is applied to separate $\beta$-decay electrons from protons, we anticipate that only $0.02 \%$ of the $\beta$-decay electrons will be misidentified as protons in the MWPC. In the UCNA project, a plastic scintillator will be implemented behind the MWPC for $\beta$-decay detection. Thus, a proton signal in the MWPC will not be miscounted as a $\beta$-decay electron.

Lastly, the energy loss and backscatter of $\beta$-decay electrons due to passage through the conversion foil was simulated. We find that only $0.12 \%$ of the incident flux is backscattered from the conversion foil with an energy greater than $50 \mathrm{keV}$ and only $0.02 \%$ is backscattered with an energy greater than $75 \mathrm{keV}$. The same simulation indicates that $99 \%$ of the transmitted $\beta$-decay electrons will lose less than $1 \mathrm{keV}, 99.9 \%$ will loose less than $3 \mathrm{keV}$, and $99.99 \%$ will loose less than $5 \mathrm{keV}$. By comparison $99.9 \%$ of the $\beta$-decay electrons will loose more than $1 \mathrm{keV}$ on passage through the MWPC.

\section{CONCLUSIONS}

The development of electron transparent, low energy proton detectors holds the promise of greatly extending the physics reach of neutron decay experiments currently designed to detect only $\beta$ particles following neutron decay. By suitably spin coating a fluorinated polyimide $(6 \mathrm{~F} 6 \mathrm{~F})$ on a silicon wafer we have been able to fabricate $600 \AA$ thick plastic substrates which can span more than $6 \times 6 \mathrm{~cm}^{2}$. At neutron decay energies, electrons are nearly unaffected by passage through such material. Perhaps more critically, these foils are substantially stronger and more durable than carbon foils used for similar applications in the past. The addition of a thin layer of $\mathrm{LiF}$ enables the conversion of $25 \mathrm{keV}$ recoil protons into $\sim 10.3$ secondary electrons, which when instrumented in a suitable high voltage stack, can be readily detected in a neutron decay spectrometer. Given the measured yield, we estimate a proton detection efficiency of nearly 97\% when instrumented in front of a simple multiwire proportional counter. Furthermore, we have constructed a small scale proton accelerator optimized for proton detector development.

As a final note, we believe that the small scale proton accelerator we have developed for the proton detector development work may be useful to others working on related problems.

\section{ACKNOWLEDGMENTS}

Thanks to Werner Tornow for his interest and support. This research was funded through DOE Grant Nos. DEFG02-97ER41033, DE-FG02-03ER41231, DE-FG0297ER41042 and NSF Grant Nos. 0100689 and 9807133. Thanks also to Bicron Inc. for the CSI crystal.

${ }^{1}$ H. Abele et al., Phys. Rev. Lett. 88, 211801 (2002).

${ }^{2}$ A. P. Serebrov et al., Sov. Phys. JETP 86, 1074 (1998).

${ }^{3}$ B. G. Erozolimskiř, Y. A. Mostovoi, V. P. Fedunin, A. I. Frank, and O. V. Khakhan, Sov. J. Nucl. Phys. 28, 48 (1978).

${ }^{4}$ A. Stolarz and P. Maier-Komor, Nucl. Instrum. Methods Phys. Res. A 480, 194 (2002).

${ }^{5}$ L. J. Lising et al., Phys. Rev. C 62, 055501 (2000).

${ }^{6}$ C. Stratowa, R. Dobrozemsky, and P. Weinzierl, Phys. Rev. D 18, 3970 (1978).

${ }^{7}$ A. L. Hallin, F. P. Calaprice, D. W. MacArthur, L. E. Piilonen, M. B. Schneider, and D. F. Schreiber, Phys. Rev. Lett. 52, 337 (1984).

${ }^{8}$ A. R. Young, C. Morris, and the UCNA Collaboration, in Fundamental Physics with Pulsed Neutron Beams, edited by C. R. Gould, G. L. Greene, F. Plasil, and W. M. Snow (World Scientific, Singapore, 2000), p. 164. 
${ }^{9}$ M. K. Ghosh and K. L. Mittal, Polyimides: Fundamentals and Applications (Marcel Dekker, New York, 1996).

${ }^{10}$ Polymers - A Property Database, edited by B. Ellis (Chapman and Hall/ CRC, New York, 2000).

${ }^{11}$ L. Zazzera and J. F. Evans, J. Vac. Sci. Technol. A 11, 934 (1993).

${ }^{12}$ D. B. Hall, P. Underhill, and J. M. Torkelson, Polym. Eng. Sci. 38, 2039 (1998).

${ }^{13}$ J. F. Ziegler, J. P. Biersack, and U. Littmark, The Stopping and Range of Ions in Solids (Pergamon, New York, 1985); the most recent version, SRIM2003 is available from www.srim.org

${ }^{14}$ J. Heidberg, in Adsorption on Ordered Surfaces of Ionic Solids and Thin Films, Springer Series in Surface Sciences Vol. 33, edited by H.-J. Freund and E. Umbach (Springer-Verlag, New York, 1993).

${ }^{15}$ N. R. Whetten, J. Appl. Phys. 35, 3279 (1964).

${ }^{16}$ J. P. Ganachaud and A. Mokrani, Surf. Sci. 334, 329 (1995).

${ }^{17}$ M. V. Gomoyunova and N. A. Letunov, Sov. Phys. Solid State 7, 316 (1965)

${ }^{18}$ A. Buzulutskov, A. Breskin, and R. Chechik, J. Appl. Phys. 81, 466 (1997).

${ }^{19}$ A. Breskin, Nucl. Instrum. Methods Phys. Res. A 371, 116 (1996).

${ }^{20}$ A. Shih, J. Yater, P. Pehrsson, J. Butler, C. Hor, and R. Abrams, J. Appl. Phys. 82, 1860 (1997).
${ }^{21}$ J. B. Miller and G. R. Brandes, J. Appl. Phys. 82, 4538 (1997).

${ }^{22}$ J. E. Yater and A. Shih, J. Appl. Phys. 87, 8103 (2000).

${ }^{23}$ H. Jahrreis and W. Oppel, J. Vac. Sci. Technol. 9, 173 (1972).

${ }^{24}$ J. Mischler, N. Benazeth, M. Negre, and C. Benazeth, Surf. Sci. 136, 532 (1984).

${ }^{25}$ B. L. Henke, J. Liesegang, and S. D. Smith, Phys. Rev. B 19, 3004 (1979).

${ }^{26}$ J. Schou, Scanning Microsc. 2, 607 (1988)

${ }^{27} \mathrm{~F}$. Sauli, in Experimental Techniques in High Energy Physics, edited by T. Ferbel (Addison-Wesley, Menlo Park, CA, 1987).

${ }^{28}$ J. Sempau, E. Acosta, J. Baró, J. M. Fernández-Varea, and F. Salvat, Nucl. Instrum. Methods Phys. Res. B 132, 377 (1997).

${ }^{29}$ B. C. Auman, in Advances in Polyimide Science and Technology: Proceedings of the Fourth International Conference on Polyimides, edited by C. Feger, M. M. Khojasteh, and M. S. Htoo (Technomic, Lancaster, PA, 1991), pp. 15-32.

${ }^{30}$ F. R. Powell, R. A. M. Keski-Kuha, M. V. Zombeck, R. E. Goddard, G. Chartas, L. K. Townsley, and J. M. Davis, in Grazing Incidence and Multilayer X-ray Optical Systems, edited by R. B. Hoover and A. B. Walker (SPIE, Bellingham, WA, 1997), Vol. 3113, pp. 432-440.

${ }^{31}$ J. Stoner, Nucl. Instrum. Methods Phys. Res. A 362, 167 (1995). 